\title{
Survey on Multimodal Biometric Authentication Combining Fingerprint and Finger vein
}

\author{
T.Sheeba, \\ Muscat College, \\ P.O.Box:2910, P.C:112, \\ Ruwi, Sultanate of Oman
}

\author{
M.Justin Bernard, \\ W.J. Towell Engineering, \\ P.O.BOX:1040, P.C: 112 \\ Ruwi, Sultanate of Oman
}

\begin{abstract}
Multimodal biometric systems have been widely used to overcome the limitations of unimodal biometric systems and to achieve high recognition accuracy. Among various multimodality options, fingerprint and finger vein has gained much attention among researchers to combine accuracy, universality and cost efficiency of the solution. This study proposes a survey of multimodal fingerprint and finger vein recognition techniques. Most of the existing work is systematically described and compared in five parts, i.e., fingerprint and finger vein image acquisition, preprocessing, feature extraction, feature matching and fusion. According to the available work in literatures and commercial utilization experiences, fingerprint and finger vein multimodality ensures higher performance and spoofing resistance. This multimodal technology has reached an unparalleled level of security, accuracy and performance.
\end{abstract}

\section{Keywords}

Biometric Authentication; Multimodal Biometrics; Fingerprint Recognition; Finger-vein Recognition

\section{INTRODUCTION}

Biometric authentication is the automated method of recognizing a person using a single biometric trait using physiological characteristics such as iris, face, fingerprints, hand geometry, handwriting, retinal, vein, and speech and/or behavioral characteristics such as voice, signature or other keystroke dynamics etc. However, recognition based on any one of these modalities may not be sufficiently robust or else may not be acceptable to a particular user group or in a particular situation or instance.

Unimodal systems that use single biometric trait for recognition purposes suffer several practical problems like non-universality, noisy sensor data, intra-class variation, restricted degree of freedom, unacceptable error rate, failureto-enroll and spoof attacks. Therefore, the performance of single biometric system need to be improved, and the techniques of multimodal biometric system can offer a feasible method to solve the problems coming from single biometric system.

Multimodal biometric system makes use of different biometric traits simultaneously to authenticate a person's identity. A comparison of popular biometrics is shown in Table 1. From the table, it is clear that combination of fingerprint and finger vein biometric traits is both an attractive alternative in comparison to other biometrics [1]. This paper investigates on the multimodal biometric authentication methods used for the fusion of two biometric traits fingerprint and finger vein.
Table 1. Comparison of parameters for different biometric technologies

\begin{tabular}{|l|l|l|l|l|l|l|l|}
\cline { 2 - 7 } \multicolumn{1}{c|}{} & \multicolumn{7}{c|}{ Biometric Parameters } \\
\hline $\begin{array}{l}\text { Biome } \\
\text { trics }\end{array}$ & $\begin{array}{l}\text { Uni } \\
\text { vers } \\
\text { alit } \\
\mathbf{y}\end{array}$ & $\begin{array}{l}\text { Uni } \\
\text { que } \\
\text { ness }\end{array}$ & $\begin{array}{l}\text { Per } \\
\text { ma } \\
\text { nen } \\
\text { ce }\end{array}$ & $\begin{array}{l}\text { Colle } \\
\text { ctabi } \\
\text { lity }\end{array}$ & $\begin{array}{l}\text { Perf } \\
\text { orm } \\
\text { anc } \\
\text { e }\end{array}$ & $\begin{array}{l}\text { Acce } \\
\text { ptabi } \\
\text { lity }\end{array}$ & $\begin{array}{l}\text { Circ } \\
\text { umv } \\
\text { enti } \\
\text { on }\end{array}$ \\
\hline Face & $\begin{array}{l}\text { Hig } \\
\mathrm{h}\end{array}$ & Low & Med & High & Low & High & Low \\
\hline $\begin{array}{l}\text { Finger } \\
\text { print }\end{array}$ & Med & $\begin{array}{l}\text { Hig } \\
\mathrm{h}\end{array}$ & $\begin{array}{l}\text { Hig } \\
\mathrm{h}\end{array}$ & Med & $\begin{array}{l}\text { Hig } \\
\mathrm{h}\end{array}$ & Med & High \\
\hline $\begin{array}{l}\text { Hand } \\
\text { try }\end{array}$ & Med & Med & Med & High & Med & Med & Med \\
\hline $\begin{array}{l}\text { Iris } \\
\mathrm{h}\end{array}$ & $\begin{array}{l}\text { Hig } \\
\mathrm{h}\end{array}$ & $\begin{array}{l}\text { Hig } \\
\mathrm{h}\end{array}$ & Med & $\begin{array}{l}\text { Hig } \\
\mathrm{h}\end{array}$ & Low & High \\
\hline $\begin{array}{l}\text { Signat } \\
\text { ure }\end{array}$ & Low & Low & Low & High & Low & High & Low \\
\hline $\begin{array}{l}\text { Voice } \\
\text { Print }\end{array}$ & Med & Low & Low & Med & Low & High & Low \\
\hline $\begin{array}{l}\text { F.Ther } \\
\text { mogra } \\
\mathrm{m}\end{array}$ & $\begin{array}{l}\text { Hig } \\
\mathrm{h}\end{array}$ & $\begin{array}{l}\text { Hig } \\
\mathrm{h}\end{array}$ & Low & High & Med & High & High \\
\hline $\begin{array}{l}\text { Retinal } \\
\text { Scan }\end{array}$ & $\begin{array}{l}\text { Hig } \\
\mathrm{h}\end{array}$ & $\begin{array}{l}\text { Hig } \\
\mathrm{h}\end{array}$ & Med & Low & $\begin{array}{l}\text { Hig } \\
\mathrm{h}\end{array}$ & Low & High \\
\hline $\begin{array}{l}\text { Vein } \\
\text { Hig }\end{array}$ & Med & Med & Med & $\begin{array}{l}\text { Hig } \\
\mathrm{h}\end{array}$ & Med & Low & \\
\hline
\end{tabular}

Fusion of fingerprint and finger vein multimodality provides the following advantages [2] like: The two biometrics are mature and independent enabling an efficient fusion, increase spoof resistance as two biometrics need to be reproduced including one which is not directly accessible, simultaneous acquisition of two sets of biometric data can be captured and processed at the same time using a single device, improves both FAR and FRR compared to mono-modal technologies used separately.

The structure of this paper is as follows. In section 2, we explain the proposed fingerprint and finger vein verification system. Section 3 presents the methods used in fingerprint and finger vein systems and Section 4 concludes the paper. 


\section{FINGERPRINT AND FINGER VEIN VERIFICATION SYSTEM}

The design of a multimodal biometric verification system consists of two stages: (1) Enrollment Phase and (2) Verification Phase. During the enrollment phase, biometric information from an individual is captured and stored. During verification phase, comparison between captured biometric and specific template stored in a biometric database is performed to verify the person's identity. Each phase has five steps: (i) Image Acquisition, (ii) Preprocessing, (iii) Feature Extraction, (iv) Classification or matching and v) Fusion. Each step is described in detail for both biometric traits fingerprint and finger vein in the following sections.

\section{METHODS}

\subsection{Image Acquisition}

\subsubsection{Fingerprint}

Two primary methods are available to capture a fingerprint image: inked (off line) and live scan (ink less) [3]. Typically an inked fingerprint image is obtained by acquiring an impression of an inked finger on a paper, and then the inked finger is scanned using a flatbed document scanner. On the other hand live scan fingerprint directly obtains fingerprint image from the sensor surface without the intermediate step of getting an impression on a paper. Various types of sensors [4] - capacitive, optical, ultrasound and thermal are used for obtaining the digital image of a fingerprint. Capacitive sensor determines each pixel value based on the capacitance measured. Optical sensors capture fingerprint images by employing optical devices like prisms to detect the change. Ultrasound sensors use ultrasonic waves and those waves may cause damage to cells on frequent exposure as they are not user-friendly. In thermal sensor pattern is produced using swiping action over the surface to measure the temperature difference.

Live scan fingerprint image is obtained based on the most popular technology [3] optical frustrated total internal reflection (FTIR) concept. There are various other live scan imaging techniques are now available, based on ultrasound total internal reflection, optical total internal reflection of edge lit holograms, thermal sensing of the temperature differential (across the ridges and valleys), sensing of differential capacitance, and noncontact three dimensional scanning.

\subsubsection{Finger vein}

Different from fingerprint, vein pattern cannot be captured by ordinary CCD camera as it is not easily seen in visible light. Instead, finger vein is captured by two ways in most existing vein recognition methods [5], a near infrared CCD sensitive camera, or an array of near infrared Light-Emitting Diode (LED). The used near infrared wavelength can be about 760 $850 \mathrm{~nm}$.

\subsubsection{Fingerprint and Finger vein}

Efficiency of multimodality is improved when two sets of biometric data are captured and processed at the same time, using a single device. Morpho [2] has launched a new range of devices that simultaneously captures, extracts and processes finger vein and fingerprint biometrics. This new multimodal technology has now reached unparalleled levels of security, accuracy and performance.

A new mobile multimodal biometric system [6] to obtain fingerprint and finger vein images simultaneously is proposed.
It uses a conventional ultra mobile personal computer (UMPC) as an embedded system for enough processing power and storage for many biometric data.

Finger vein verification machine made by HITACHI is used in [4] and this device is made multimodal by fixing a small capacitive sensor over its device thereby making both fingerprint and finger vein device operate in at same time to capture their respective images.

[7] proposed a device which acquires fingerprint and a fingervein image at the same time from the first and second knuckles of finger, respectively. The device's size is so small that can be adopted it on a mobile device, easily.

\subsection{Pre-Processing}

\subsubsection{Fingerprint}

Once the input fingerprint images is captured, it is necessary to preprocess the images inorder to improve the clarity of ridge structures of fingerprint images by maintaining their integrity, remove the noise and blurring effect and retain the connectivity of the ridges while maintaining separation between ridges.

The fingerprint images go through preprocessing stage in which operations like Normalization [8][9], Binarization [10][11][12], Segmentation methods like block direction estimation[10],direction variety check [10], morphological operations 'OPEN' and 'CLOSE'[10] [12], skin colour detection, adaptive thresholding, morphological processing[9], pixel-wise method and block-wise method like contextual filtering based on short-time fourier transform (STFT), discrete cosine transform (DCT) and K-means [13] etc.

Enhancement methods like appropriately tuned Gabor filter [8], STFT analysis [9],histogram Equalization and Fourier Transform [10], fuzzy set[11], spatial domain method, histogram equalization, and contrast limited adaptive histogram equalization [12], Gabor filters [13] for smoothing out noise, discrete wavelet transform (DWT), singular value decomposition (SVD) and fuzzy measures [14] etc.

Image Thinning [10] [11], Image Compression and Image Restoration etc.

\subsubsection{Finger vein}

After the raw image captured, it is required to be preprocessed before feature extraction. In the preprocessing of finger vein image [5], the samples are usually first sent for image enhancement. Next, all the samples are cut into moderate dimension images of the same size, usually called region of interest (ROI). Next, these biometric samples are reduced to mathematical templates and only these enrolled temples are stored in system database. Obviously, a template size should be applicable (e.g., 256 or 512 bytes) in order to alleviate the burden of limited storage space.

Finger vein undergone preprocessing operations like normalization [15], two-dimensional normalization [16] using the outline of the finger, high frequency enhanced filtering [17] to enhance filtering image, In [18] circular gabor filter to enhance finger-vein region, then image segmentation for finger-vein network extraction, then thinning to obtain the finger-vein skeleton, [19] performs normalization, orientation image estimation, gabor filtering, image segmentation and image thinning. Segmentation of the finger region, histogram stretching and brightness normalization are performed in [20]. 


\subsection{Feature Extraction}

Feature extraction involves representing the image by a set of numerical features to remove redundancy from the data and reduce its dimension.

\subsubsection{Fingerprint}

Feature extractions on fingerprint recognition are mainly based on four methods: minutiae based methods, wavelet based approach, statistical approaches and fractal approach methods.

Minutiae based methods: The minutia points are classified into four classes namely termination, bifurcation, trifurcation, crossovers etc. Various methods based on the minutiae based representation were proposed in [12] which uses morphological operation to extract minutiae, [10] uses thinning and crossing number to extract minutiae, [11] uses crossing number method to extract minutia points, an extraction algorithm [21] which examines the neighborhood pixels around each pixel of the thinned ridges is used to extract the minutiae, gabor filter [9] [22] is used for feature extraction, a midpoint ridge contour [8] is used as an efficient alternative of minutiae extraction for fingerprint images without using thinning operation, algorithm based on Tico and Kuosmanen method and crossing number methods used in [11] to extract minutia points. The main disadvantages [3] in minutia based methods are: it needs more computational complexity due to the preprocessing operations on fingerprint images like image enhancement, directional filtering, ridge segmentation, ridge thinning and minutiae extraction. Moreover the fingerprint images with low quality (low resolution or low illumination) cannot provide them for reliable minutiae extraction.

Optical wavelet approach Haa's wavelet [24] used as a feature extractor has achieved limited ability to track the position, shift, rotation, scale and intensity invariance. Besides, the clusters obtained from the wavelet features have more overlap region thus reducing the ability to separate out the clusters during the matching stage of the recognition process.

Statistical approach uses fingerprint localization algorithm followed by Pseudo Zernike Moments Invariant (PSMI) [23] for feature extraction. It includes two more parameters like fingerprint candidate threshold (FPCT) and axis correction ratio (ACR) for efficient and robust feature extraction which classify all fingerprints in the database with a $100 \%$ accuracy.

Hybrid approach [25] combines both PZM and wavelets extracts most significant features from the fingerprint images and achieve better verification rate.

Fractal approach [3] achieves high recognition rates as well as low computational complexity and independence from variation of scale and rotation and has facilities in obtaining different depth information for the same image. It has achieved an efficient solution for a small fingerprint recognition system.

\subsubsection{Finger vein}

Nowadays, many works have been done for finger-vein based personal identification, and extracting features related to the finger-vein network is a common way in these works. Conventional methods such as the matched filter and morphological [28] methods can extract patterns if the widths of veins are constant.

Feature extractions on finger vein recognition are mainly based on approaches like local vein shape approach, wavelet and curvelet based approach and holistic vein approach.
Local vein shape [16] uses line tracking that starts from various positions and achieves equal error rate of $0.145 \%$ in personal identification. [26] extracts global finger vein patterns by iteratively tracking local lines from various positions to robustly extract finger vein patterns from unclear images. In [27] matched-filter method similar to a ridgelet function is used to extract the feature mode of finger-vein. [28] extract the centerlines of the veins consistently without being affected by the fluctuations in vein width and brightness, so its pattern matching is highly accurate.

[29] proposed wavelet combined with principal component analysis (PCA) transformation and LDA transformation for finger vein recognition. Wavelet transform [15] is adopted to extract the veins from the captured images.

However, these current methods [18] are always sensitive to finger-vein image qualities, since the noises caused by processing low contrast finger-vein images can greatly reduce their stability and reliability in real applications. In addition the recognition accuracy can also be reduced. This method also requires considerable processing time for finger vein extraction.

[17] explains process of finger vein feature extraction process which achieves $99 \%$ accuracy. [30] proposed a multiscale feature extraction method of finger-vein patterns based on curvelets and local interconnection structure neural networks to extract features of finger-vein pattern and proved to extract features efficiently from obscure images.

A novel method [18] used to exploit finger-vein features of local moments, topological structure and statistics of finger veins were extracted. In [19] minutiae features, bifurcation points and ending points are extracted from the vein patterns which is used as a geometric representation of the vein patterns shape. An enhanced method [31] for extracting finger vein feature based on morphology which combines morphological peak and valley detection is proposed. [32] introduced a new method of finger vein extraction using gradient normalization, curvature calculation, and binarization.

Finger vein extraction that uses holistic vein texture does not perform finger vein extraction so they need less processing time. In [33] local binary pattern (LBP) is extracted from the normalized image. [34] proposed a method of reducing the positional disparities of the vein images with the minutiae of vein matching based on LBP. [20] proposed a new identification method of finger vascular patterns using weighted LBP codes based on a support vector machine (SVM).

\subsection{Classification and Matching}

\subsubsection{Fingerprint}

Classification is the most important stage of the system. Various methods [13] have been proposed for classification: rule-based, syntactic-based, structure-based, statistical-based, neural-network based and multi-classifier approach.

Some of the widely used classifiers are two stage classifier KNearest Neighbour followed by Support Vector Machines [13], Fuzzy Logic measures [13], f u z z y evolutionary program m ing [ 113 ], Principal component analysis [35], Neural networks [8] [11] [12] [21] [23] [24], Neural network and genetic algorithm [36] etc. [37] analyzed existing classifiers and concluded that the usage of neural network as classifier will provide better security than other techniques. 
The fingerprint feature matching algorithms are usually quite similar for both fingerprint verification and identification. Fingerprint matching approach is categorized into four, correlation-based matching, minutiae-based matching, pattern matching or ridge feature based technique, and image based matching [10].

There are various algorithms used for fingerprint matching. Some of these algorithms are point based matching, string distance-based minutiae matching algorithm, hough transform-based minutiae matching, constraint satisfaction based technique [38], alignment based greedy matching algorithm and support vector classifier [39], minutia score matching method [40] etc.

General distance measures or classifiers used for the comparison purpose in verifying the identity of the individual through feature matching [13] are absolute distance, manhattan distance, hamming distance, euclidean distance, cosine angle and fuzzy closeness of membership function [3] etc.

\subsubsection{Finger vein}

There are several popular used approaches of similarity measurement used for finger vein matching. Some of the methods adopted for finger vein matching are template matching [16] [28], nearest cosine classifier [18], modified hausdorff distance [19] [20], hausdorff distance [15] and weighted match score [30] etc.

\subsection{Fusion}

Fusion of multimodal biometrics comes under four levels [41]: Sensor level, feature level, decision level and matching score level. In sensor level fusion, biometric traits taken from sensors are combined and processed to form a composite biometric trait. In feature level fusion, biometric traits coming from different biometric channels are first preprocessed, and then feature vectors are extracted separately using specific fusion algorithm and these feature vectors are combined to form a composite feature vector which is then used for classification process. In decision level fusion, each modality is first pre-classified independently. The outputs of the different modalities are then fused together in the final classification. In matching score level fusion, the feature vectors are processed separately and individual matching score is found, then depending on the accuracy of each biometric channel the matching level is fused to find composite matching score which will be used for classification.

An image level fusion [4] is applied to both finger print and finger veins using intersection points considered as identification factor. This multimodal system proved better security and lower error rates in finger recognition.

A feature level fusion of fingerprints and finger vein biometrics is proposed in [42]. Fingerprints and finger vein are first preprocessed separately, then minutiae's are extracted, and then feature level fusion is done by concatenating the feature points obtained from fingerprint and finger vein. Finally, weight matching algorithm is proposed to match the feature points with the query images.

Decision level fusion is proposed in [7] to touched fingerprint and finger-vein. Second-level decision fusion algorithm [43] based on fingerprint and finger vein dual-mode recognition is performed which proved improvement in the recognition performance of the system.
Score level fusion [44] of fingerprint finger vein recognition is proposed and experimental results demonstrate dramatically improvement in performance.

\section{CONCLUSION}

This study presents a survey of multimodal fingerprint and finger vein recognition techniques for biometric authentication and identification. It presents the general framework, key techniques, available methods available for the multimodal fingerprint and finger vein technology. According to the available work in literatures and commercial utilization experiences, fingerprint and finger vein multimodality ensures higher performance, spoofing resistance, unparalleled level of security and accuracy.

\section{REFERENCES}

[1] Hatim A. Aboal samh, "A Multi Biometric System Using Combined Vein and Fingerprint Identification", International Journal of Circuits, Systems and Signal Processing, pp 29-36, Issue 1, Volume 5, 2011.

[2] The VP Series: The First Ever Multimodal Finger vein and Fingerprint Devices, "http://www.ifsec.co.uk/ExhibitorLibrary/135/Triptyque _HD_3.pdf”, accessed June 2012.

[3] Chaitanya Kommini, Srinivasulu Asadi, Kamalesh Ellanti,"Scale and Rotation Independent Fingerprint Recognition", (IJCSIT) International Journal of Computer Science and Information Technologies, 17641773, Vol. 2 (4), 2011.

[4] Arunkumar.V, Malathy.C, "MULTIMODAL BIOMETRICS BY FUSION OF FINGER VEIN AND FINGER PRINT IMAGES", Proceedings of the 4th National Conference; INDIACom-2010.

[5] Hao Luo, Fa-Xin Yu, Jeng-Shyang Pan, Shu-Chuan Chu and Pei-Wei Tsai," A Survey of Vein Recognition Techniques" Information Technology Journal 9(6): 1142-1149, ISSN 1812-5638, 2010.

[6] H.C Lee, K.R Park, B.J Kang, S.J Park, “A New Mobile Multimodal Biometric Device Integrating Finger Vein and Fingerprint Recognition”, IEEE,2009.

[7] Y.H Park, D.N Tien, H.C Lee, K.R Park, E.C Lee, S.M Kim, H.C Kim,"A Multimodal Biometric Recognition of Touched Fingerprint and Finger-vein", International Conference on Multimedia and Signal Processing, IEEE,2011

[8] Bhupesh Gour, T.K.Bandopadhyaya, Sudhir Sharma," Fingerprint Feature Extraction Using Midpoint ridge Contour method and Neural Network", IJCSNS International Journal of Computer Science and Network Security, Vol.8 No.7, July 2008.

[9] B.Y. Hiew, Andrew B.J. Teoh and Y.H. Pang, "Digital Camera based Fingerprint Recognition", IEEE International conference on Telecommunications and Malaysia International Conference on Communications, pp.676-680, 2007.

[10] M.Kaur, M.Singh, A.Girdhar, and P.S. Sandhu, "Fingerprint verification System using Minutiae Extraction Technique", World Academy of Science, Engineering and Technology,46, pp 497-502, 2008. 
[11] Pravesh Kumar, "Use of Fuzzy Set and Neural Network to Extract Fingerprint Minutiae Points and Location", Thesis, 2009.

[12] Atanu Chatterjee, Shuvankar Mandal, G.M.Atiqur Rahaman and Abu Shamim Mohammed Arif,'Fingerprint Identification and Verification System by Minutiae Extraction Using Artificial Neural Network", JCIT, ISSN 2078-5828 (PRINT), ISSN 2218-5224 (ONLINE), Volume 01, Issue 01, 2010.

[13] B. Shanmuga Priya, R. Rajesh," A Note on Fingerprint Recognition Systems", 978-1-4244-8679-3/11/, IEEE, 2011.

[14] D.Bennet and Dr. S. Arumuga Perumal, "Fingerprint: DWT, SVD Based Enhancement and Significant Contrast for Ridges and Valleys Using Fuzzy Measures" Journal of Computer Science and Engineering, Volume 6, Issue 1, March 2011.

[15] Li Xueyan Guo Shuxu Gao Fengli, Li Ye," Vein Pattern Recognitions by Moment invariants", IEEE, 2007.

[16] N.Miura, A.Nagasaka, T.Miyatake,"Feature extraction of finger-vein patterns based on repeated line tracking and its application to personal identification", Machine Vision and Applications, pp 194-203, 2004.

[17] Z. Lian, Z. Rui and C. B. Yu, "Study on the Identity Authentication System on Finger Vein", in International Conference on Bioinformatics and Biomedical Engineering. IEEE, pp.1905-1907, 2008.

[18] Jinfeng Yang, Yihua Shi, Jinli Yang, Lihui Jiang," A NOVEL FINGER-VEIN RECOGNITION METHOD WITH FEATURE COMBINATION”, IEEE, 2009.

[19] Cheng-Bo Yu, Hua-Feng Qin, Lian Zhang, Yan-Zhe Cui, "Finger-vein image recognition combining modified hausdorff distance with minutiae feature matching", $J$. Biomedical Science and Engineering, 2, 261-272, 2009.

[20] Hyeon Chang LEE1, Byung Jun KANG2, Eui Chul LEE3, Kang Ryoung PARK," Finger vein recognition using weighted local binary pattern code based on a support vector machine", Journal of Zhejiang UniversitySCIENCE C (Computers \& Electronics), 11(7):514-524, 2010.

[21] Md. Mamunur Rashid and Aktar Hossain, A.K.M., "Fingerprint Verification System Using Artificial Neural Network", (ISSIN 1812-5638) Information Technology Journal 5(6): 1063-1067, 2006.

[22] Dhruv Batra, Girish Singhal and Santanu Chaudhury, "Gabor Filter based Fingerprint Classification using support Vector Machines", IEEE India Annual conference, pp.256-260, 2004.

[23] Idris. E.-Feghi, Adel. Tahar, Hosain Aboasha, Zhijie Xu," Efficient Features Extraction for Fingerprint Classification with Multi Layer Perceptron Neural Network", $8^{\text {th }}$ International Multi-Conference on Systems, Signals \& Devices, 2011.

[24] M.ANTOWIAK and K.CHALASINSKAMACUKOW,"Fingerprint identification by using artificial neural network with optical wavelet preprocessing", OPTO-ELECTRONICS REVIEW 11(4), $327-337,2003$
[25] Avinash Pokhriyal, Sushma Lehri," A new method of fingerprint authentication using 2D wavelets", Journal of Theoretical and Applied Information Technology, Vol. 13 No.2 March, pp (131 - 138), 2010.

[26] Naoto Miura, Akio Nagasaka, Takafumi Miyatake, "Feature Extraction of Finger Vein Patterns Based on Iterative Line Tracking and Its Application to Personal Identification", System and Computers in Japan, vol. 35 , No. 7, 2004.

[27] M. Kono, H. Ueki, and S. Umemura, "Near-Infrared Finger Vein Patterns for Personal Identification," Applied Optics, vol. 41, pp. 7429-7436, 2002.

[28] N. Miura, A. Nagasaka and T. Miyatake, "Extraction of Finger Vein Patterns Using Maximum Curvature Points in Image Profiles", IEICE-Transactions on Information and Systems, pp.185-1194, 2007.

[29] K. Wang and Z. Yuan, "Finger Vein Recognition Based on Wavelet Moment Fused with PCA Transform", Pattern Recognition and Artificial Intelligence (in Chinese). Vol.20, No.5, pp.692-697, 2007.

[30] Z. Zhang, S. Ma and X. Han, "Multiscale Feature Extraction of Finger-Vein Patterns Based on Curvelets and Local Interconnection Structure Neural Network", in International Conference on Pattern Recognition. IEEE, pp.145-148, 2006.

[31] MEI Cong-li, XIAO Xiao, LIU Guo-hai, CHEN Yu, LI Qian-an, "Feature Extraction of Finger-vein Image Based on Morphologic Algorithm", Sixth International Conference on Fuzzy Systems and Knowledge Discovery, IEEE, 2009.

[32] Choi, J.H., Song, W.S., Kim, T.J., Lee, S.R., Kim, H.C., "Finger vein extraction using gradient normalization and principal curvature. SPIE, 7251:1-9, 2009.

[33] Jang, Y.K., Kang, B.J., Park, K.R., "A study on touchless finger vein recognition robust to the alignment and rotation of finger". KIPS Trans. Part B, 15-B (4):275284, 2008.

[34] Lee, E.C., Lee, H.C., Park, K.R., "Finger vein recognition by using minutia based alignment and local binary pattern- based feature extraction". Int. J. Imag. Syst. Technol., 19(3):179-186, 2009.

[35] Ching-Tang Hsieh, Shys-Rong Shyu, "Principal Component analysis for Minutiae Verification on Fingerprint Image", Proc. Of International Conference on Multimedia Systems \& Signal Processing”, pp.45-49, 2007.

[36] LI Xiangrong WANG Guohui, LU Xiangjiang, "Neural Network Based Automatic Fingerprints Classification Algorithm" IEEE, 2010.

[37] P.M.Gomathi, Dr.G.M. Nasira,"A Survey on Biometrics based Key Authentication using Neural Network" Global Journal of Computer Science and Technology" Volume 11, Issue 11, Version 1.0, July 2011.

[38] Florina Muntenescu, "Fingerprint Matching on Small Images", STUDIA UNIV. BABES_BOLYAI, INFORMATICA, Volume LIV, Number 1, 2009. 
[39] Jianjiang Feng, "Combining minutiae descriptors for fingerprint matching" Pattern Recognition, 342 - 352, 2008.

[40] RAVI. J, K. B. RAJA, VENUGOPAL. K. R, "FINGERPRINT RECOGNITION USING MINUTIA SCORE MATCHING", International Journal of Engineering Science and Technology, Vol.1(2), 35-42, 2009.

[41] Ashish Mishra,"Multimodal Biometrics it is: Need for Future Systems", International Journal of Computer Applications (0975 - 8887), Volume 3 - No.4, June 2010 .
[42] AKunming Lin, Fengling Han, Yongming Yang and Zulong Zhang," Feature Level Fusion of Fingerprint and Finger Vein Biometrics", Springer-Veriag Berlin Heidelberg, pp. 348 - 355, 2011.

[43] WANG Ke-jun, MA Hui, LI Xue-feng,"Research on dual-modal second-level decision fusion for fingerprint and finger vein recognition", Control and Decision, Vol. 26 No. 8, Aug.2011.

[44] Feifei CUI, Gongping YANG," Score Level Fusion of Fingerprint and Finger Vein Recognition", Journal of Computational Information Systems, 5723-5731, December, 2011. 